\title{
A Golden Opportunity: Prevention in the Workplace
}

\author{
R. $\operatorname{Volpe}^{1} \cdot$ S. Marchant ${ }^{2}$
}

Received: 27 July 2015/Accepted: 8 September 2015/Published online: 16 September 2015

(C) Springer International Publishing Switzerland 2015

Notwithstanding prevention campaigns geared at improving the lifestyle habits of our patients and the availability of continually improved pharmaceuticals, cardiovascular diseases remain the number one cause of death in the western world.

One of the main reason for this relatively failure is unsatisfactory risk factor control. In fact, the findings of the Cardiovascular Epidemiology Observatory (OEC) Project of the Italian National Institute of Health (ISS) and the National Centre for Disease Prevention and Control (CCM) of the Italian Ministry of Health, which compared the prevalence of the main cardiovascular risk factors in the period 2008-2012 with those of 1998-2002, show that while data on blood pressure and smoking has improved, data on cholesterol and body weight has worsened, with an increased prevalence of an overweight and obese population. Moreover, even if there has been an improvement of the percentage of patients in treatment for diabetes, high cholesterol and hypertension, the majority (i.e. $59 \%$ individuals with diabetes and hypertension and $72 \%$ of those with high cholesterol), are not aware of their risk factor, or if they are, do not seek treatment. Lastly, simply because patients are receiving medical care, this does not mean their condition is under control; we often observe

R. Volpe

roberto.volpe@cnr.it

S. Marchant

smarchant@unccd.int

1 Health and Safety Office, Italian National Research Council, Rome, Italy

2 Staff Association Executive Committee, United Nations Convention to Combat Desertification, Bonn, Germany incomplete medication adherence and as a consequence, patients to not achieve the desired results prescribed by the medical treatment guidelines. As a result, only about $20 \%$ of patients with diabetes, high cholesterol and hypertension receive appropriate, satisfactory medical care. The Italian data reflect the results of the European Action on Secondary and Primary Prevention by Intervention to Reduce Events III (EUROASPIRE III), conducted in 22 European countries in 2006-2007, that show that a large proportion of coronary patients do not achieve the desired therapeutic targets for cardiovascular risk factors. Moreover, the European Study on Cardiovascular Risk Prevention and Management in Daily Practice (EURIKA), conducted in 12 European countries in 2009-2010, concludes that a lack of control of hypertension, hyperlipidemia, diabetes and smoking was responsible for $30 \%$ of cardiovascular mortality risk.

This bleak picture is an alarm bell for us to improve cardiovascular prevention strategies at local, national and international levels, including seeking new ways of interaction with professional figures-not necessarily experts in prevention-who, once sensitized and trained, can help improve this picture. This approach, for instance, can also be adopted in the workplace, in the context of "workplace medicine", which has the major advantage of constant, "close" interaction between medical practitioners, and employees who are at risk. Examples of this are the studies conducted in Rome by the Italian National Research Council's Prevention and Safety Department (CNR-SPP) on CNR's working population (the "PRO.SA.", or Health Promotion Project) and the Coronary Heart Disease Prevention Programme of the Medical Service of the Food and Agriculture Organization of the United Nations (FAO). Both studies screened for the prevalence of risk factors and 
made computerized calculations of the cardiovascular risk level, in collaboration with CNR and FAO's medical service, proposed dietary and nutraceutical intervention in the first instance, but also pharmacological intervention, where necessary. This approach has led to a marked improvement in risk factors, with a significant reduction in the level of cardiovascular risk.

This is why, in the workplace too, it is our duty to cooperate with occupational health practitioners to increase their awareness and motivate them, so that employees are steered towards healthier lifestyles and, when receiving medical care, adhere more closely to the prescribed treatment. These aspects are the key to enhancing cardiovascular prevention. That this is a step in the right direction has been reinforced by the European Heart Network that at the request the Italian Society for Cardiovascular Prevention (SIPREC) and the Italian Heart Foundation has put workplace medicine on the standing agenda of its annual meeting. 